\title{
Avaliação das competências andragógicas dos docentes do nível técnico de enfermagema
}

\author{
Assessment of the andragogical skills of teachers at the level nursing technician \\ Evaluación de las competencias andragógicas de los professores del nível técnico de enfermeira
}

Rejane Faria Habyak Prado ${ }^{1,2}$ (1) Noélle de Oliveira Freitas ${ }^{2}$ (D)

1. Serviço Nacional de Aprendizagem

Comercial. São Paulo, SP, Brasil.

2. Universidade Guarulhos, Programa de

Pós-Graduação em Enfermagem. Guarulhos, SP, Brasil.
Autor correspondente:

Noélle de Oliveira Freitas.

E-mail: nooliveirafreitas@gmail.com.

Recebido em 09/09/2020.

Aprovado em 20/01/2021.

\section{Resumo}

Objetivos: Identificar como o enfermeiro docente do nível técnico de enfermagem avalia suas competências andragógicas como facilitador de aprendizagem, para o exercício da docência. Método: Estudo transversal com abordagem quantitativa, desenvolvido com 73 enfermeiros docentes, em 30 unidades de uma instituição de nível técnico de enfermagem do Estado de São Paulo. Os dados foram coletados no período de setembro a dezembro de 2019. Foi aplicada a Escala de Classificação Autodiagnóstica de Competências para o Papel do Educador/Instrutor de Adultos. Foi utilizado teste Wilcoxon. Resultados: Amostra predominantemente feminina $(72,6 \%)$, com média de idade de 40,3 anos ( $D P=7,4)$ e tempo de atuação docente de $9,7$ anos ( $\mathrm{DP}=6,3)$. Os docentes consideraram que seu nível atual de competência encontra-se em moderado conhecimento para o desenvolvimento de cada competência, enquanto o nível que desejava atuar com adultos em processos de ensino $e$ aprendizagem deveria estar em alto conhecimento. Conclusão e implicações para a prática: Os docentes consideraram-se abaixo do nível desejado, em todos os itens avaliados nas competências andragógicas, como facilitadores de aprendizagem para o exercício da docência. O estudo apresenta grande relevância de inovação, pois não há disponível na literatura qualquer estudo que avalie as competências andragógicas dos docentes do nível técnico de enfermagem.

Palavras-chave: Competência Profissional; Docentes de Enfermagem; Educação Profissionalizante; Educação em Enfermagem; Enfermagem

\section{Abstract}

Objective: To identify how the teaching nurse of the technical level of nursing evaluates his/her andragogical skills as a facilitator of learning, for the exercise of teaching. Method: A cross-sectional study with a quantitative approach, developed with 73 teaching nurses, in 30 units of a technical nursing level institution in the State of São Paulo. The data was collected from September to December 2019. The Self-diagnostic Classification Scale of Competencies for the Educator of Adults Role was applied. Wilcoxon's test was used. Results: A predominantly female sample (72.6\%), with a mean age of 40.3 years $(\mathrm{SD}=7.4)$ and teaching time of 9.7 years $(S D=6.3)$. The teachers considered that their current level of competence is in moderate knowledge for the development of each skill, while the level that they wanted to work with adults in teaching and learning processes should be in high knowledge. Conclusions and implications for practice: The teachers considered themselves below the desired level, in all the items evaluated in the andragogical skills, as learning facilitators for the teaching exercise. The study presents great innovation relevance, because there is not available in the literature any study that evaluates the andragogical competences of the teachers of the technical nursing level.

Keywords: Professional Competence; Faculty, Nursing; Education, Professional; Education, Nursing; Nursing.

\section{Resumen}

Objetivo: Identificar cómo el enfermero docente del nivel técnico de enfermería evalúa sus habilidades andragógicas como facilitador del aprendizaje para el ejercicio de la enseñanza. Método: Estudio descriptivo transversal con enfoque cuantitativo, desarrollado con 73 enfermeras docentes en 30 unidades de una institución de nivel técnico de enfermería en el Estado de São Paulo. Los datos se recogieron de septiembre a diciembre de 2019. Se aplicó la Escala de calificación de aptitudes de autodiagnóstico para la función de educador/instructor de adultos. Se utilizó la prueba de Wilcoxon. Resultados: Muestra predominantemente femenina $(72,6 \%$ ), con una edad media de 40,3 años ( $D E=7,4)$ y experiencia docente de 9,7 años $(D E=6,3)$. Los docentes consideraron que su nivel actual de competencia es de conocimiento moderado para el desarrollo de cada competencia mientras que el nivel que deseaba trabajar con adultos en los procesos de enseñanza y aprendizaje debía ser de conocimiento alto. Conclusiones e implicaciones para la práctica: Los profesores se consideraron por debajo del nivel deseado, en todos los ítems evaluados en las competencias andragógicas, como facilitadores de aprendizaje para el ejercicio de la docencia. $\mathrm{E}$ estudio presenta gran relevancia teniendo como característica la innovación, ya que no existe ningún estudio disponible en la literatura que evalúe las competencias andragógicas de los profesores de enfermería a nivel técnico.

Palabras clave: Competencia Profesional; Docentes de Enfermería; Educación Profesional; Educación en Enfermería; Enfermería. 


\section{INTRODUÇÃO}

O termo andragogia tem origem nos termos gregos andros (homem adulto), agein (conduzir) e logos (ciência), ou seja, uma ciência com o objetivo de conduzir os adultos ao aprendizado. Esse termo foi utilizado pela primeira vez, em 1833, pelo pedagogo alemão Alexander Kapp que o empregou para descrever a teoria educacional do filósofo grego Platão. ${ }^{1}$ Posteriormente, o termo andragogia foi utilizado por um cientista social alemão, Eugen Rosenstock, em um relatório para Academia do Trabalho de Frankfurt, em $1921^{1}$ Eugen Rosenstock afirmou que a educação de adultos exigia professores, métodos e uma filosofia especial, não bastava apenas transpor a teoria sobre educação, baseada na pedagogia, para a realidade dos adultos. ${ }^{1}$

A partir da década de 1970, Malcolm Knowles começou a utilizar o termo andragogia para nomear a educação com adultos, de acordo com a concepção de que adultos e crianças aprendem de maneiras diferentes. Os adultos, diferentemente das crianças, possuem a necessidade de tomar suas próprias decisões, muitas vezes inquietando-se com imposições que vão contra seu anseio de se autodirigir. Os profissionais que atuam como docentes de adultos, ao se deparar com essa característica, empenham-se em propiciar experiências de aprendizagem nas quais os alunos adultos são apoiados a se tornarem aprendizes autodirigidos e não mais dependentes. ${ }^{1}$ Desde então, as ideias de Malcolm Knowles têm sido citadas em estudos relacionados à Andragogia. ${ }^{2-6}$

Sendo assim, a andragogia é um conjunto de princípios de aprendizagem de adultos, sendo estes, a necessidade do aprendiz de saber, o autoconceito do aprendiz, a experiência anterior do aprendiz, a prontidão para aprender, a orientação para aprendizagem e a motivação para aprender. ${ }^{1}$ Para atuar na educação com adultos o docente deve desenvolver determinadas competências e habilidades distintas da atuação na educação com crianças e adolescentes.

No contexto atual, é relevante olhar para o docente e para as competências que este deve deter, pois as escolas que promovem uma boa formação são representadas por indivíduos que desejam ensinar (docentes) e outros que desejam aprender (alunos). ${ }^{7}$

A competência tem vários significados, entre os quais a qualidade de quem é capaz de apreciar e resolver determinado assunto, de produzir determinada coisa ou possuir uma capacidade, habilidade ou aptidão, ou seja, de maneira geral, a noção de competência se apresenta sempre associada a ação. ${ }^{8}$ Neste sentido, o conceito da competência vem sendo enfaticamente empregado com o objetivo de associar o conhecimento teórico à prática, tendo como centro, o indivíduo capaz de tal realização. ${ }^{8}$ Uma das abordagens sobre competências mais difundida é usualmente conhecida como $\mathrm{CHA}$ (Conhecimentos, Habilidade e Atitudes). O "Conhecimento" diz respeito ao domínio intelectual da área de atuação, do conhecimento, da informação, entendimento claro e correto. A "Habilidade" é definida como a capacidade de saber fazer, da aplicação, da técnica e da experiência. E as
"Atitudes" relacionam-se ao comportamental e à capacidade de tomar decisões adequadas a cada situação, ou seja, saber ser. ${ }^{9}$

Para avaliar as competências do docente na educação com adultos, em 1981, Malcolm Knowles publicou o instrumento "Escala de Classificação Autodiagnóstica de Competências para o Papel do Educador/Instrutor de Adultos" baseado em três categorias de competências que o professor/educador deveria desenvolver para trabalhar com adultos: facilitador da aprendizagem, desenvolvedor e administrador de programas educativos para adultos. ${ }^{1}$ Essa escala foi utilizada em estudos para avaliar a competência dos docentes, em cursos de graduação em Enfermagem. ${ }^{7,10,11}$

Um estudo realizado com 226 docentes de cursos de graduação, no município de São Paulo, utilizou a Escala de Classificação Autodiagnóstica de Competências para o Papel do Educador/Instrutor de Adultos. Neste estudo, concluiu-se que a maioria dos docentes enfermeiros encontrava-se distante do nível máximo de competência desejada, além disso, identificouse a necessidade de mensurar previamente o nível "atual" de competência dos docentes, antes de propor a implementação da andragogia como fundamento para a formação de professores. ${ }^{7}$

Entre os profissionais da enfermagem $75,6 \%$ pertencem ao nível técnicos segundo o relatório do Conselho Federal de Enfermagem de 2020. ${ }^{12}$ Para atuar na docência no nível técnico pela natureza tecnicista da educação profissionalizante, muitas vezes o docente que atua nessa área não é visto como profissional da educação, porém para que este consiga realizar um trabalho de boa qualidade, é necessário ter conhecimento específico da profissão e conhecimentos pedagógicos para exercer a função de docente. ${ }^{13}$

O docente que atua na formação de nível médio deve ter, principalmente, a experiência profissional como enfermeiro, e o preparo para o magistério será realizado em serviço, em cursos de licenciatura ou em programas especiais de formação pedagógica, pois em educação profissional quem ensina deve saber fazer, principalmente na educação em enfermagem, ou seja, ter o conhecimento da prática e não apenas o conhecimento teórico. $\mathrm{E}$, além disso, quem sabe fazer e quer ensinar deve aprender a ensinar. ${ }^{14}$

Dessa forma, ressalta-se que o presente estudo tem como característica a inovação, posto que não há na literatura qualquer estudo que aborde sobre as competências andragógicas dos docentes de enfermagem do nível técnico. Estudos relacionados a esta temática foram desenvolvidos somente com docentes de cursos graduação em enfermagem, ${ }^{7,10,11}$ entre outros cursos da área da saúde foi identificado um estudo com docentes do curso de medicina que teve como objetivo avaliar as competências pedagógicas sob a perspectiva do aluno. ${ }^{15}$ Uma revisão integrativa publicada em 2017 identificou somente seis estudos publicados entre 2001 a 2013 que abordam sobre as competências necessárias ao docente na graduação em enfermagem. Entre os estudos identificados na revisão, os resultados apontaram como competências essenciais aos enfermeiros docentes a comunicação, a habilidade pedagógica, a educação continuada, 
a ética e a humanização, além de compreender o tempo e os sentimentos do aluno, constituir vínculos, respeitar e reconhecer a autonomia de cada um. ${ }^{16}$

Acredita-se que os resultados deste estudo poderão contribuir para o planejamento de programas de desenvolvimento de docentes e de instituições de ensino de nível técnico, no tocante às competências importantes para melhorar o desempenho de docentes e discentes. Sendo assim, justifica-se a realização desta pesquisa.

Portanto, o objetivo deste estudo foi identificar como o enfermeiro docente do nível técnico de enfermagem avalia suas competências andragógicas como facilitador de aprendizagem, para o exercício da docência.

\section{MÉTODO}

Estudo descritivo, de corte transversal, com abordagem quantitativa, realizado em 30 unidades do Serviço Nacional de Aprendizagem Comercial (Senac), sendo três na capital, três na região metropolitana e 24 em cidades do interior do Estado de São Paulo.

A população elegível para este estudo foi composta pelos 271 docentes que atuavam nas unidades do Senac. O convite foi enviado a todos, tendo aceitado a participar da pesquisa e respondido aos questionários $73(26,9 \%)$ docentes. Os critérios de inclusão do estudo foram enfermeiros docentes que atuam na função ministrando aulas teóricas e/ou práticas, sendo excluídos enfermeiros docentes que estavam de férias ou em afastamento, durante o período da coleta dos dados.

Os docentes foram abordados por meio do e-mail institucional no qual foi enviado um convite para participação no estudo com orientações sobre seus objetivos e os procedimentos. Os dados foram coletados no período de setembro a dezembro de 2019.

Os instrumentos foram respondidos on-line pelo Google Forms ${ }^{\circledR}$. Para acessar os questionários, o participante acessou um link enviado por e-mail. Ao acessar este link, o participante teve acesso inicialmente ao Termo de Consentimento Livre e Esclarecido (TCLE). Ao acessar o TCLE, o participante assinalava no link se aceitaria ou não participar da pesquisa. Após aceitar, os sujeitos acessaram os instrumentos: o questionário de caracterização da amostra e a Escala de Classificação Autodiagnóstica de Competências para o Papel do Educador/Instrutor de Adultos. ${ }^{1}$

A Escala de Classificação Autodiagnóstica de Competências para o Papel do Educador/Instrutor de Adultos foi construída em 1981 por Malcolm Knowles. Trata-se de uma escala de de autoavaliação sobre as competências atuais e desejadas do docente para atuar com adultos, em processos de ensino e aprendizagem. A escala completa é composta por 55 itens distribuídos em três categorias de competências que o professor/ educador deveria desenvolver para trabalhar com adultos: facilitador da aprendizagem, desenvolvedor e administrador de programas educativos para adultos, totalizando 55 itens. ${ }^{1} \mathrm{O}$ presente estudo utilizou as competências como "Facilitador da Aprendizagem", composta por 29 itens e duas questões dissertativas. dividos em quatro domínios: I - Estrutura conceitual e teórica da aprendizagem de adultos (5 itens), II - Design e aplicação de experiências de aprendizagem (7 itens), III - Auxiliar aprendizes a serem autodirigidos (3 itens) e IV - Selecionar métodos, técnicas e materiais (14 itens). Cada item descreve a habilidade do docente em escala tipo Likert, de 0 a 5 [0 - ausente, 1 - muito baixo conhecimento, 2 - baixo conhecimento, 3 - moderado conhecimento (compreensão conceitual), 4 - alto conhecimento e 5 - altíssimo conhecimento (expert ou especialista)]. O sujeito deve escrever a letra "A" na opção em que considera como o nível atual de desenvolvimento para cada competência, e a letra "R" deve corresponder ao ponto em que desejava estar atuando com adultos em processos de ensino e aprendizagem. ${ }^{1}$

Os dados obtidos por meio do Google Forms ${ }^{\circledR}$ foram transcritos para um banco de dados construído com a ferramenta Microsoft Excel®. Após construído o banco, os dados da planilha foram transportados para software Stata ${ }^{\circledR}$ versão 15.0. O nível de significância adotado para todos os testes foi de 0,05.

A primeira etapa das análises constituiu-se de análise descritiva das informações sociodemográficas e profissionais dos docentes. Foram calculadas medidas de posição (média e mediana) e medidas de dispersão (desvio-padrão, mínimo e máximo). A categorização das informações, necessária para aplicação dos testes de diferença de médias, também foi realizada, e as frequências foram calculadas.

A segunda etapa constitui-se na análise das pontuações dos níveis atual e desejado para cada questão pertencente aos cinco domínios da competência facilitador da aprendizagem. Para tanto, foram inicialmente calculadas medidas de posição e dispersão. Posteriormente, as pontuações de cada nível, em cada questão, foram analisadas para verificação. Neste teste, considera-se que a distribuição é significativamente a diferença da distribuição normal, quando o valor de $p$ for inferior a 0,05 Portanto, distribuições normais são aquelas com valor de $p$ superior a 0,05. Todas as distribuições analisadas apresentaram valor de $p$ inferior a 0,05 , permitindo concluir, portanto, que possuíam distribuição não normal. A análise de resultados do teste Shapiro-Wilk levou à escolha do teste não paramétrico de Wilcoxon para comparar os resultados do nível atual e do nível desejado, para cada pergunta.

Em seguida, os mesmos parâmetros de posição e dispersão foram calculados, dessa vez para os domínios e considerando os níveis atual e desejado. Mais uma vez a distribuição dos resultados foi testada com o teste Shapiro-Wilk, e de novo seu resultado indicou o uso do teste não paramétrico dos sinais de Wilcoxon, para identificar a existência de diferenças estatisticamente significantes $(p<0,05)$, entre os resultados do nível atual e do nível desejado em cada domínio.

O estudo foi aprovado pelo Comitê de Ética em Pesquisa da Universidade Univeritas UNG Guarulhos (Protocolo CAAE: 13324819.2.0000.5506; Número do Parecer: 3.520.027). Foram obedecidas as orientações constantes da Resolução 466/12 do Conselho Nacional de Saúde, em relação aos aspectos éticos com a instituição e com os sujeitos que participaram da pesquisa. ${ }^{17}$ 


\section{RESULTADOS}

Dos 73 docentes, 53 (72,6\%) eram do sexo feminino, 51 $(69,9 \%)$ tinham entre 31 e 45 anos, 51 (69,5\%) trabalhavam de 30 a 40 horas semanais e $67(91,8 \%)$ ministravam aulas teóricas e estágios. O tempo de atuação como docente variou de 1 a 38 anos, com média de 9,7 anos (DP =6,3), já o tempo de atuação como docente do Senac teve variação entre 1 e 21 anos, com média de 5,7 anos (DP = 4,4). Em relação à formação, 50 (68,5\%) eram especialistas, sendo $32(43,8 \%)$ especialistas em docência (Tabela 1).

Os resultados dos itens da Escala de Classificação Autodiagnóstica de Competências para o Papel do Educador/ Instrutor de Adultos mostraram que, em relação à competência "Facilitador da aprendizagem", os docentes apresentaram maior média $(3,7 ; \mathrm{DP}=0,9)$ para o nível de competência atual $(A)$, no item 3 ("Habilidade para criar um ambiente físico e psicológico no qual respeito, confiança, sinceridade, apoio e segurança sejam mútuos") do domínio II (Design e aplicação de experiências de aprendizagem). Em relação ao nível de competência desejada (D), os docentes apresentaram maior média no valor de
4,7 (DP = 0,7), no item 12 ("Habilidade para inventar novas técnicas de maneira a adaptá-las a novas situações") do domínio IV (Selecionar métodos, técnicas e materiais) (Tabela 2).

Em todos os itens de avaliação do nível atual de competência os docentes consideraram que a encontravam-se na média de 2 a 3 pontos (baixo a moderado conhecimento) na Escala de Classificação Autodiagnóstica de Competências para o Papel do Educador/Instrutor de Adultos. Ao avaliarem o nível desejado de competência, os docentes pontuaram em média acima de 4 (alto conhecimento), em todos os itens da escala. Todos os resultados de comparação entre a pontuação atual e a desejada da competência foram estatisticamente significantes $(p<0,05)$ (Tabela 2).

Na Tabela 3, estão apresentados os resultados da avaliação da competência "Facilitador de aprendizagem", por domínios. Os valores das médias do nível de competência atual $(A)$ e desejado (D) foram próximos. Para o nível de competência atual (A), os domínios II "Design e aplicação de experiências de aprendizagem" e IV "Selecionar métodos, técnicas e materiais" apresentaram a maior média de 3,3 (DP =0,8), nos dois

Tabela 1. Distribuição dos sujeitos do estudo, segundo as variáveis sociodemográficas ( $\mathrm{n}=73$ ). Estado de São Paulo, Brasil, 2019.

\begin{tabular}{|c|c|c|c|c|c|c|}
\hline Variáveis & $\mathbf{N}$ & (\%) & Média & (DP)* & Mediana & (Intervalo) \\
\hline \multicolumn{7}{|l|}{ Sexo } \\
\hline Feminino & 53 & $(72,6)$ & & & & \\
\hline Masculino & 20 & $(27,4)$ & & & & \\
\hline Idade & 73 & $(100)$ & 40,3 & $(7,4)$ & 40,7 & $(27,2-60,8)$ \\
\hline Tempo de formação (em anos)* & 73 & $(100)$ & 12,6 & $(5,7)$ & 12,0 & $(3,0-38,0)$ \\
\hline Pós-graduação & 73 & $(100)$ & & & & \\
\hline Especialização & 50 & $(68,5)$ & & & & \\
\hline Mestrado & 15 & $(20,5)$ & & & & \\
\hline Doutorado & 5 & $(6,8)$ & & & & \\
\hline Pós-doutorado & 2 & $(2,7)$ & & & & \\
\hline Não possui & 1 & $(1,4)$ & & & & \\
\hline Especialização em Docência & 32 & $(43,8)$ & & & & \\
\hline Tempo de atuação como docente (em anos) & 73 & $(100)$ & 9,7 & $(6,3)$ & 9,0 & $(1,0-38,0)$ \\
\hline $\begin{array}{l}\text { Tempo de atuação como docente no Senac } \\
\text { (em anos) }\end{array}$ & 73 & $(100)$ & 5,7 & $(4,4)$ & 5,0 & $(1,0-21,0)$ \\
\hline
\end{tabular}

Atuação docente

Aulas teóricas e estágios

$67(91,8)$

Somente aulas teóricas

$6 \quad(8,2)$

Atua como enfermeiro em instituição de saúde

\begin{tabular}{|c|c|c|c|c|c|c|}
\hline Sim & 58 & $(79,5)$ & & & & \\
\hline Não & 15 & $(20,5)$ & & & & \\
\hline Carga horária semanal no Senac & 73 & $(100)$ & 31,7 & $(7,8)$ & 32,0 & $(6,0$ a 40,0$)$ \\
\hline
\end{tabular}

*Desvio-padrão 
Tabela 2. Descrição dos resultados da competência Facilitador da aprendizagem, por itens, segundo docentes do curso técnico de enfermagem $(n=73)$. Estado de São Paulo, Brasil, 2019.

\begin{tabular}{|c|c|c|c|c|c|c|c|c|}
\hline Domínios & Item & Nível & Média & (DP)* & Mediana & Mínimo & Máximo & $p$-valor** \\
\hline \multirow{10}{*}{$\begin{array}{c}\text { (I) Estrutura } \\
\text { conceitual e teórica } \\
\text { da aprendizagem de } \\
\text { adultos }\end{array}$} & \multirow{2}{*}{1} & $A^{* * *}$ & 3,0 & 0,9 & 3 & 0 & 5 & \multirow{2}{*}{0,000} \\
\hline & & $\mathrm{D} * * * *$ & 4,4 & 0,8 & 4 & 1 & 5 & \\
\hline & \multirow{2}{*}{2} & $A$ & 2,9 & 1,0 & 3 & 0 & 5 & \multirow{2}{*}{0,000} \\
\hline & & D & 4,3 & 0,9 & 5 & 1 & 5 & \\
\hline & \multirow{2}{*}{3} & A & 3,1 & 0,9 & 3 & 0 & 5 & \multirow{2}{*}{0,000} \\
\hline & & D & 4,4 & 0,8 & 5 & 1 & 5 & \\
\hline & \multirow{2}{*}{4} & A & 3,0 & 1,1 & 5 & 0 & 3 & \multirow{2}{*}{0,000} \\
\hline & & D & 4,4 & 0,8 & 5 & 1 & 5 & \\
\hline & \multirow{2}{*}{5} & A & 3,5 & 1,0 & 4 & 0 & 5 & \multirow{2}{*}{0,000} \\
\hline & & D & 4,5 & 0,8 & 5 & 1 & 5 & \\
\hline \multirow{14}{*}{$\begin{array}{l}\text { (II) Design e aplicação } \\
\text { de experiências de } \\
\text { aprendizagem }\end{array}$} & \multirow{2}{*}{1} & $A$ & 2,8 & 1,2 & 3 & 0 & 5 & \multirow{2}{*}{0,000} \\
\hline & & D & 4,3 & 0,9 & 5 & 1 & 5 & \\
\hline & \multirow{2}{*}{2} & $A$ & 3,2 & 0,9 & 3 & 1 & 5 & \multirow{2}{*}{0,000} \\
\hline & & D & 4,4 & 0,9 & 5 & 1 & 5 & \\
\hline & \multirow{2}{*}{3} & A & 3,7 & 0,9 & 4 & 1 & 5 & \multirow{2}{*}{0,000} \\
\hline & & D & 4,6 & 0,8 & 5 & 1 & 5 & \\
\hline & \multirow{2}{*}{4} & A & 3,7 & 0,9 & 4 & 1 & 5 & \multirow{2}{*}{0,000} \\
\hline & & D & 4,6 & 0,8 & 5 & 1 & 5 & \\
\hline & \multirow{2}{*}{5} & A & 3,4 & 0,9 & 4 & 1 & 5 & \multirow{2}{*}{0,000} \\
\hline & & D & 4,5 & 0,8 & 5 & 1 & 5 & \\
\hline & \multirow{2}{*}{6} & A & 3,3 & 1,0 & 3 & 0 & 5 & \multirow{2}{*}{0,000} \\
\hline & & D & 4,5 & 0,9 & 5 & 1 & 5 & \\
\hline & \multirow{2}{*}{7} & A & 3,2 & 1,0 & 3 & 0 & 5 & חמח \\
\hline & & $\mathrm{D}$ & 4,5 & 0,8 & 5 & 1 & 5 & 0,000 \\
\hline & 1 & A & 3,0 & 1,0 & 3 & 0 & 5 & م000 \\
\hline & 1 & D & 4,4 & 0,9 & 5 & 1 & 5 & 0,000 \\
\hline (III) Auxiliar aprendizes & 2 & A & 3,1 & 1,0 & 3 & 0 & 5 & חم ח \\
\hline a serem autodirigidos & 2 & $\mathrm{D}$ & 4,4 & 0,9 & 5 & 1 & 5 & 0,000 \\
\hline & 3 & $A$ & 3,0 & 1,0 & 3 & 0 & 5 & חת ח \\
\hline & 3 & $\mathrm{D}$ & 4,5 & 0,8 & 5 & 1 & 5 & 0,000 \\
\hline & 1 & A & 3,1 & 0,9 & 3 & 0 & 5 & חמחת \\
\hline & 1 & $\mathrm{D}$ & 4,5 & 0,8 & 5 & 1 & 5 & 0,000 \\
\hline & 2 & A & 3,3 & 1,0 & 3 & 0 & 5 & חמת מ \\
\hline $\begin{array}{l}\text { (IV) Selecionar } \\
\text { métósnicas }\end{array}$ & 2 & D & 4,6 & 0,8 & 5 & 1 & 5 & 0,000 \\
\hline materiais & 3 & A & 3,4 & 1,0 & 4 & 1 & 5 & חمח ח \\
\hline & 3 & D & 4,6 & 0,7 & 5 & 1 & 5 & 0,000 \\
\hline & 1 & A & 3,1 & 0,9 & 3 & 1 & 5 & م000 \\
\hline & 4 & D & 4,5 & 0,8 & 5 & 1 & 5 & 0,000 \\
\hline
\end{tabular}

*Desvio-padrão; ${ }^{* *}$ Teste de Wilcoxon; ${ }^{* * *}$ Atual; ${ }^{* * * *}$ Desejado 
Tabela 2. Continuação

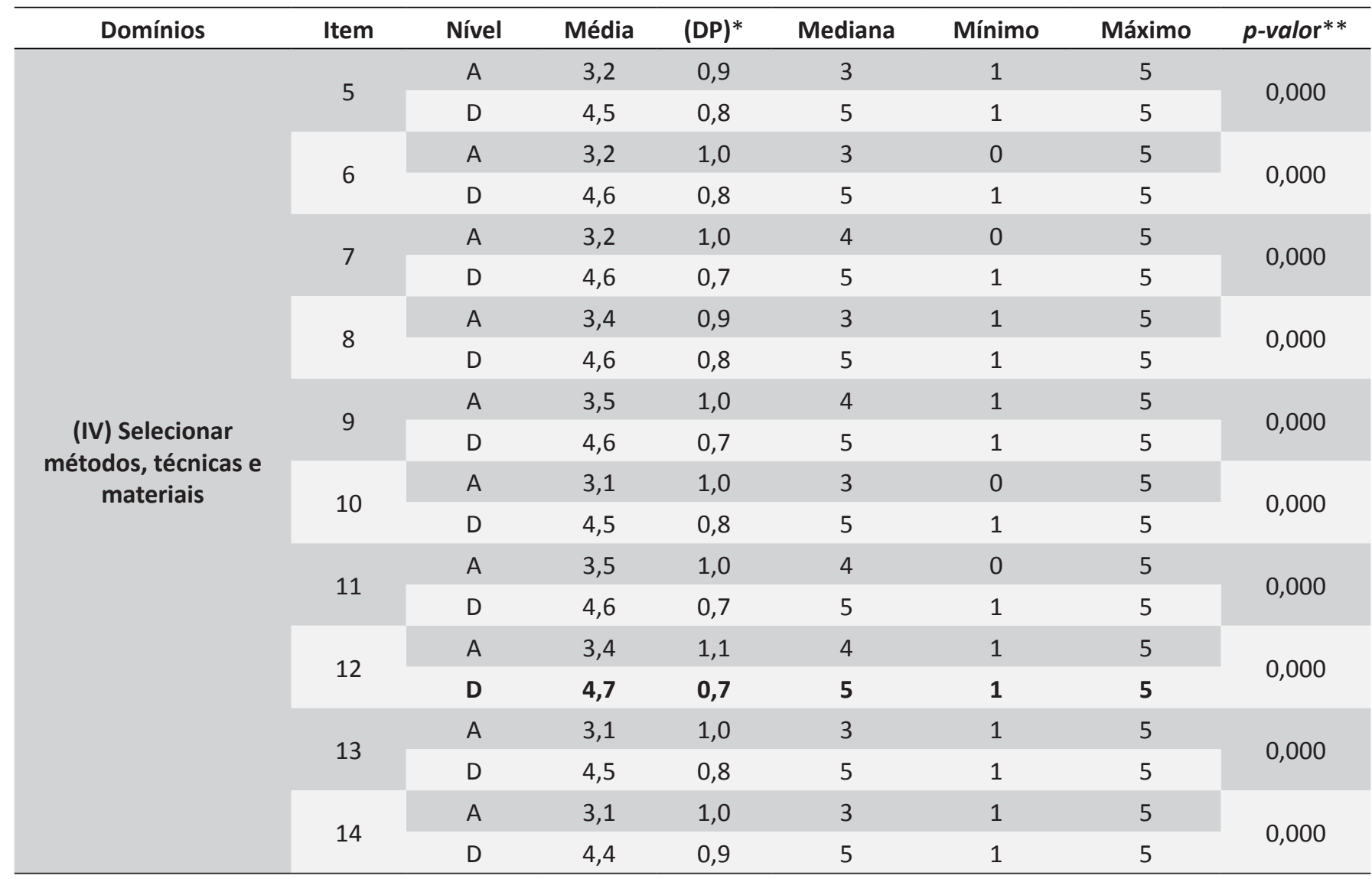

*Desvio-padrão; ${ }^{* *}$ Teste de Wilcoxon; ${ }^{* * *}$ Atual; ${ }^{* * * *}$ Desejado

Tabela 3. Descrição dos resultados da competência "Facilitador da aprendizagem", por domínio, segundo docentes do curso técnico de enfermagem ( $n=73$ ). Estado de São Paulo, Brasil, 2019.

\begin{tabular}{lcccccc}
\hline \multicolumn{1}{c}{ Domínios } & Nível & Média & (DP)* & Mediana & Mínimo & Máximo \\
\hline (I) Estrutura conceitual e teórica da & A $^{* *}$ & 3,1 & 0,9 & 3,2 & 0 & 5 \\
aprendizagem de adultos & D $^{* * *}$ & $\mathbf{4 , 4}$ & $\mathbf{0 , 8}$ & $\mathbf{4 , 8}$ & $\mathbf{1}$ & $\mathbf{5}$ \\
(II) Design e aplicação de & $\mathrm{A}$ & $\mathbf{3 , 3}$ & $\mathbf{0 , 8}$ & $\mathbf{3 , 4}$ & $\mathbf{0 , 9}$ & $\mathbf{5}$ \\
experiências de aprendizagem & $\mathrm{D}$ & 4,5 & 0,8 & 4,9 & 1 & 5 \\
(III) Auxiliar aprendizes a serem & $\mathrm{A}$ & $\mathbf{3 , 0}$ & $\mathbf{0 , 9}$ & $\mathbf{3 , 0}$ & $\mathbf{0}$ & $\mathbf{5}$ \\
autodirigidos & $\mathrm{D}$ & $\mathbf{4 , 4}$ & $\mathbf{0 , 8}$ & $\mathbf{5 , 0}$ & $\mathbf{1}$ & $\mathbf{5}$ \\
\hline (IV) Selecionar métodos, técnicas e & $\mathrm{A}$ & $\mathbf{3 , 3}$ & $\mathbf{0 , 8}$ & $\mathbf{3 , 4}$ & $\mathbf{0 , 9}$ & $\mathbf{5}$ \\
materiais & $\mathrm{D}$ & $\mathbf{4 , 6}$ & $\mathbf{0 , 9}$ & $\mathbf{0 , 7}$ & $\mathbf{1}$ & $\mathbf{5}$ \\
\hline
\end{tabular}

*Desvio-padrão; ** Atual; *** Desejado

domínios. Já o domínio III “Auxiliar aprendizes a ser autodirigidos” apresentou menor média para o nível de competência atual (A) 3,0 (DP =0,9). Para o nível de competência desejado (D), o domínio IV "Selecionar métodos, técnicas e materiais" apresentou maior média 4,6 (DP =0,9). Menores médias foram identificadas nos domínios I "Estrutura conceitual e teórica da aprendizagem de adultos" e III "Auxiliar aprendizes a ser autodirigidos", no valor de 4,4 (DP =0,8).
Na Figura 1, observou-se associação estatisticamente significativa entre as médias dos domínios de competência desejada entre os docentes $(p=0,028)$.

\section{DISCUSSÃO}

No que diz respeito à caracterização da amostra, identificouse uma população predominantemente feminina $(72,6 \%)$ e com 

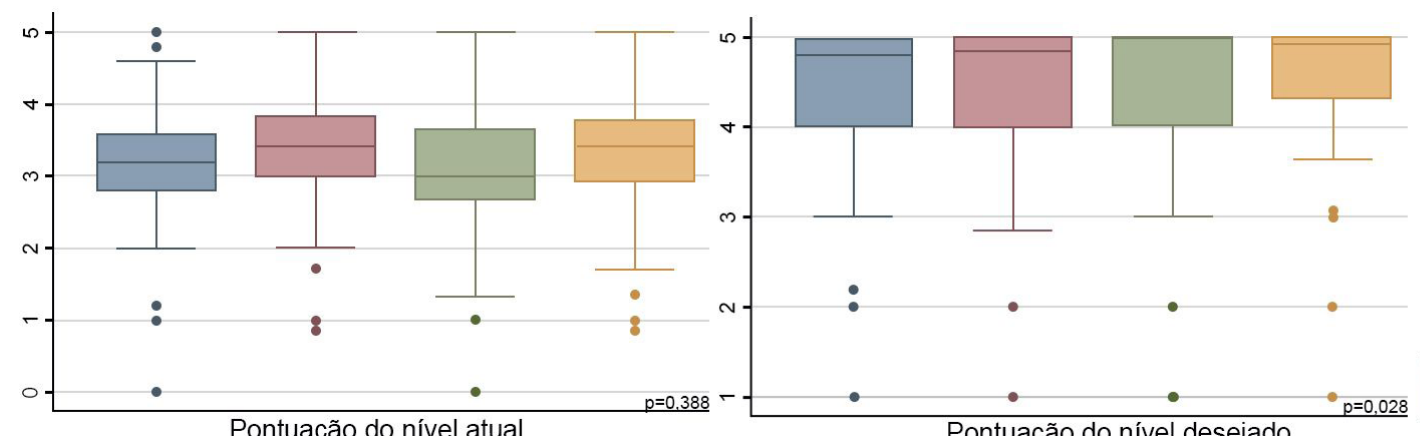

Pontuação do nível desejado

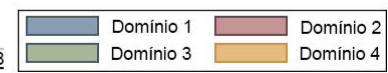

Figura 1. Box plot de comparação das médias dos domínios da competência "Facilitador de Aprendizagem" considerando o nível de competência atual e o desejado, segundo os docentes do curso técnico de enfermagem ( $n=73$ ). Estado de São Paulo, Brasil, 2019.

idade entre 31 e 45 anos. A maioria com pós-graduação, dos quais $43,8 \%$ com especialização em docência, com média de tempo de atuação como docente de 9,7 anos (DP =6,3), o tempo de atuação como docente na Instituição com média de 5,7 anos (DP $=4,4), 67(91,8 \%)$ atuavam em aulas teóricas e práticas e $58(79,5 \%)$ atuavam na área assistencial em instituições de saúde. Um estudo, realizado com docentes enfermeiros de graduação em enfermagem, teve comparativamente com a amostra do presente estudo a maioria dos participantes do sexo feminino que atuava tanto em aulas teóricas como práticas e trabalhava em carga horária de 30 a 40 horas semanais, porém no que diz respeito à formação dos docentes que atuam no nível superior, observou-se uma porcentagem maior de especialistas, mestres e doutores. . $^{70,11}$ Sendo assim, evidencia-se que os docentes na área da enfermagem, principalmente aqueles que participaram deste estudo que atuam no nível médio, tem um perfil de experiência na docência sendo majoritariamente especialistas, atuando em aulas teóricas e práticas e com experiência e conhecimento da prática. Este perfil profissional geralmente é recomendado para atuação em um curso de nível médio, principalmente no tocante a experiência na prática, pois é relevante que o docente tenha conhecimento da prática para ensinar os alunos de um curso técnico de enfermagem. Um estudo que avaliou a percepção de estudantes de medicina sobre as competências docentes identificou a necessidade relatada pelos estudantes de aliar a teoria e a prática para se obter um processo ensino-aprendizagem de qualidade ressaltando que atividades práticas eficazes podem contribuir para a assimilação do conhecimento teórico. ${ }^{15}$ Dessa forma, evidencia-se que a importância do conhecimento prático do docente que atua em cursos da área da saúde, principalmente em cursos técnicos, pois as atividades práticas aproximam o aluno da dinâmica de funcionamento das instituições de saúde e da realidade biopsicossocial do paciente contribuindo para a melhoria da formação. ${ }^{15}$

A análise dos resultados da Escala de Classificação Autodiagnóstica de Competências para o Papel do Educador/ Instrutor de Adultos permitiu identificar que houve diferença estatisticamente significante $(p<0,001)$, entre o nível de competência atual e o nível desejado em todos os itens. No domínio I "Estrutura conceitual e teórica da aprendizagem de adultos", identificou-se que o item 5 ("Habilidades para conceituar e explicar o papel do professor como facilitador e fonte de recursos para os aprendizes autodirigidos") foi o item no qual os docentes consideraram ter maior habilidade, assim como maior interesse em desenvolver-se. O item 2 ("Habilidades para descrever as diferenças de premissas sobre os jovens e adultos como aprendizes e as implicações dessas diferenças para o ensino") evidenciou menor habilidade e menor necessidade de desenvolver-se.

O docente que atua na educação de adultos deve compreender que a proposta da andragogia se trata de um processo de aquisição de competências e autodireção do aluno. ${ }^{1}$ No entanto, em geral os docentes são treinados e aprendem por meio da pedagogia e fundamentam as suas atividades na docência pautadas em modelos que tiveram quando alunos, sendo assim não são conduzidos a desenvolverem habilidades de acordo com a concepção andragógica ${ }^{18}$ e o uso de recursos metodológicos. ${ }^{7}$ Além disso, o aluno também não está preparado para assumir a autodireção e desenvolver uma atitude autodirigida, pois ao longo da sua trajetória acadêmica também aprendeu sob o fundamento da pedagogia. ${ }^{\top}$

No o domínio III "Auxiliar os aprendizes a ser autodirigidos", os docentes consideraram que o item 1 ("Habilidades para explicar a diferença conceitual entre instrução didática e aprendizagem autodirigida") era a habilidade que menos desejavam desenvolver, e o item 3 ("Habilidades para modelar o papel de aprendizagem autodirigida em seu próprio comportamento") obteve a menor pontuação no nível atual de competência e a maior no nível desejado. Nesse domínio, identificou-se que o item no qual os docentes desejam alcançar um nível mais alto de competência é aquele no qual se avaliam menos capacitados. Algumas competências relacionadas aos aspectos da andragogia, da aprendizagem autodirigida, apresentaram menor pontuação, embora 43,8\% dos docentes que participaram do estudo eram especialistas em docência, isso ressalta que mesmo com experiência em docência e especialização na área os docentes ainda têm dificuldade em trabalhar com a aprendizagem autodirigida. Segundo a teoria da Aprendizagem Autodirigida (Self-Directed 
Learning), os adultos são aprendizes autodirigidos. ${ }^{1}$ Aprendizes adultos tendem a querer assumir a responsabilidade própria, no planejamento, na implementação e na avaliação de suas atividades de aprendizagem. ${ }^{1}$

No domínio IV "Selecionar métodos, técnicas e materiais", identificaram-se os itens 10 ("Habilidade para usar com eficácia métodos de participação de audiência") e 4 ("Habilidades para provar uma análise racional para selecionar um método, uma técnica ou um material em particular, para alcançar objetivos educacionais específicos"), como menores níveis atuais de competência. No item 4 , foi identificada, também, a menor pontuação desejada. Os docentes afirmaram ter maior domínio do item 9 ("Habilidade para usar com eficácia vários métodos experimentais e simulações"), assim como maior necessidade de desenvolver o item 12 ("Habilidade para inventar novas técnicas de maneira a adaptá-las a novas situações"). No decorrer da carreira acadêmica, os docentes desenvolvem mais habilidades para trabalhar com conteúdos teóricos, pois é um aspecto valorizado pela pedagogia. De modo geral, o enfoque no processo ensino e aprendizagem e o uso de recursos metodológicos não são habituais na prática de docentes que aprenderam a valorizar conteúdos teóricos ${ }^{7}$ Dessa forma, para os docentes que atuam no nível médio torna-se ainda mais desafiador desenvolver habilidades para trabalhar com adultos considerando a educação profissional no qual é relevante ter o conhecimento da prática e, com isso, deve-se saber fazer e ensinar. ${ }^{14}$

Comparativamente aos resultados do estudo com os docentes de graduação em enfermagem, ${ }^{7,10,11}$ após a análise dos quatro domínios presentes na competência "Facilitador da aprendizagem", identificou-se que os docentes do nível técnico avaliaram-se mais habilitados em todos os itens que compõem os quatro domínios, assim como os docentes do nível superior demonstraram maior desejo de desenvolvimento em 24 dos 29 itens presentes.

Em determinadas competências, houve similaridades na autoavaliação dos docentes do nível técnico e dos docentes de graduação em enfermagem, as quais puderam ser identificadas nos domínios I "Estrutura conceitual e teórica da aprendizagem de adultos" e II "Design e aplicação de experiências de aprendizagem"7,10,11 Essa convergência traz a reflexão de que algumas habilidades podem ser tanto para os docentes que atuam no nível técnico como para aqueles que atuam no nível superior. As divergências foram evidenciadas nos domínios III "Auxiliar aprendizes a ser autodirigidos" e IV "Selecionar métodos, técnicas e materiais". Existem algumas especificidades que poderiam justificar essas divergências, como o fato de que, no nível técnico, o docente em geral ministra diversas disciplinas e tem um olhar mais tecnicista, tendo como característica docente o "saber fazer", devido aos fazeres profissionais inerentes ao técnico em enfermagem. No nível superior, os docentes em geral são responsáveis por ministrar disciplinas específicas, já que o profissional enfermeiro necessita de conhecimentos mais aprofundados para sua atuação e exerce a função de supervisão dos estágios nos serviços de saúde, tendo, portanto, a necessidade de uma formação de maior complexidade.
Uma revisão de literatura que investigou a produção científica sobre Andragogia nas Ciências da Saúde, no período de 1999 a 2009, identificou que a área da Enfermagem foi responsável por um maior volume de publicações sobre essa temática. ${ }^{19}$ Os resultados demonstram que a Enfermagem é uma área com grande interesse na temática Andragogia e que isso pode estar relacionado ao aspecto de que tradicionalmente aborda o educar como uma das ferramentas para o exercício da profissão. ${ }^{19}$

Embora a pesquisa realizada no formato online possibilite uma facilidade na coleta de dados por vários fatores, como a comodidade do participante em responder ao questionário no domicílio de forma virtual, o que poderia resultar no aumento de respostas recebidas, pois o pesquisador não tem o trabalho, o tempo e o dinheiro despendido em imprimir e aplicar os questionários pessoalmente,,$^{20,21}$ no entanto, esta forma de coleta de dados possui limitações como as questões individuais dos participantes no uso de ferramentas eletrônicas, a exclusão dos analfabetos digitais e a impossibilidade do conhecimento das circunstâncias em que o questionário deve ser respondido. ${ }^{21}$

Essas limitações podem acarretar baixa taxa de retorno dos questionários, a literatura relata que a taxa de retorno de questionários é em torno de $25 \%{ }^{15}$ e alguns estudos identificaram uma taxa de $25,2 \%$ a $67 \% .{ }^{20,22,23}$ Neste estudo, a taxa de retorno foi de $26,9 \%$, mantendo-se dentro da faixa prevista pela literatura. ${ }^{20,22,24}$

\section{CONCLUSÕES E IMPLICAÇÕES PARA A PRÁTICA}

Em geral, os participantes deste estudo afirmaram encontrarse em um nível moderado de competência, em todos os itens avaliados, já no nível desejado a maioria relatou que desejaria estar em um nível mais elevado de conhecimento. Em relação aos domínios, os participantes declararam maior desenvolvimento atual nos domínios "Design e aplicação de experiências de aprendizagem" "Selecionar métodos, técnicas e materiais". Já, em relação ao desejo de desenvolver-se, apontaram o domínio "Selecionar métodos, técnicas e materiais" como aspecto que ainda necessita ser desenvolvido. Sendo assim, os resultados sugerem que o nível de competência atual dos docentes não é tão elevado quanto o nível de competência que desejavam encontrar-se, o que sugere que os docentes reconhecem que necessitam desenvolver mais competências para atuar na educação de adultos no nível médio de enfermagem e alcançar o nível de competência que almejam. Como limitação deste estudo destaca-se a escassez de outros estudos que relacionem as competências andragógicas de docentes de enfermagem de formação de nível técnico, o que dificulta a comparação e discussão dos resultados, sendo então feita a comparação com os resultados de estudos realizados com docentes de enfermagem do ensino superior e de outras áreas. Além disso, outra limitação que merece ser comentada é a amostragem aleatória utilizada na presente pesquisa. Este tipo de amostragem pode acarretar um possível viés na pesquisa, pois os docentes que aceitaram participar do estudo podem ser aqueles que se consideram mais competentes. 
No entanto, o presente estudo apresenta relevância que merece ser comentada como a possibilidade de ser utilizado como base para a condução de estudos futuros com docentes do nível técnico de enfermagem. Estudos futuros poderão desenvolver intervenções nos processos de formação do docente para desenvolvimento individual e institucional com o objetivo de melhorar o desempenho dos docentes como cursos sobre temas de ensino e aprendizagem voltados para a educação com adultos, o que irá impactar na melhoria do ensino para os alunos do nível técnico de enfermagem.

\section{CONTRIBUIÇÕES DOS AUTORES}

Desenho do estudo. Rejane Faria Habyak Prado. Noélle de Oliveira Freitas.

Coleta ou produção dos dados. Rejane Faria Habyak Prado.

Análise de dados. Rejane Faria Habyak Prado. Noélle de

Oliveira Freitas.

Interpretação dos resultados. Rejane Faria Habyak Prado.

Noélle de Oliveira Freitas.

Redação e revisão crítica do manuscrito. Rejane Faria Habyak Prado. Noélle de Oliveira Freitas

Aprovação da versão final do artigo. Rejane Faria Habyak Prado. Noélle de Oliveira Freitas.

Responsabilidade por todos os aspectos do conteúdo e a integridade do artigo publicado. Rejane Faria Habyak Prado. Noélle de Oliveira Freitas.

\section{EDITOR ASSOCIADO}

\section{Gerson Luiz Marinho}

\section{REFERÊNCIAS}

1. Knowles MS, Holton 3rd EF, Swanson RA. Aprendizagem de resultados: uma abordagem prática para aumentar a efetividade da educação corporativa. $2^{\mathrm{a}}$ ed. Rio de Janeiro: Elsevier; 2011

2. Botma Y, Seale I, Spies C. Adult learning: What nurse educators need to know about mature students. Curationis. 2015;38(2):1494. http:// dx.doi.org/10.4102/ curationis.v38i2.1494. PMid:26842085.

3. Hamilton B, Leigh K, Whitted K. Integration of Andragogy into Preceptorship. J. Adult Educ. 2015;42(1):9-17.

4. Dalby D, Norrie P. How adult are our learners? A cross-sectional exploration of the learning characteristics of nursing students in a United Kingdom University. J Res Nurs. 2007;12(4):319-29. http:// dx.doi.org/10.1177/1744987107075254.

5. Jinks AA. Applying Education Theory to Nursing Curricula: nurse teachers' definitions of student-centred andragogical teaching and learning concepts. J Furth High Educ. 2006;23(2):221-30. http://dx.doi. org/10.1080/0309877990230206.

6. Loeng S. Various ways of understanding the concept of andragogy. Loeng. Cogent Education. 2018;5(1):1496643. http://dx.doi. org/10.1080/2331186X.2018.1496643.
7. Draganov PV, Sanna MC. Avaliação das competências dos professores de enfermagem para facilitar a aprendizagem de adultos. Cogitare Enferm. 2015;20(3):556-64. http://dx.doi.org/10.5380/ce.v20i3.41104.

8. Pinhel I, Kurcgant P. Reflexões sobre competência docente no ensino de enfermagem. Rev Esc Enferm USP. 2007;41(4):711-6. http://dx.doi. org/10.1590/S0080-62342007000400024. PMid:18193629.

9. Banov MR. Recrutamento, Seleção e Competências. São Paulo: Atlas 2015. p.28.

10. Draganov PV, Sanna MC. Avaliação das competências dos professores de enfermagem para administrar programas educativos para adultos. Esc Anna Nery.2014;18(1):167-74. http://dx.doi.org/10.5935/1414-8145.20140025.

11. Draganov PV, Sanna MC. Competências andragógicas dos docentes enfermeiros que atuam na graduação em enfermagem paulistana. Trab Educ Saúde. 2016;14(1):155-82. http://dx.doi.org/10.1590/1981-7746sip00098.

12. Conselho Federal de Enfermagem. Enfermagem em números [Internet] Brasília: COFEN;2020 [citado 2020 Dez 01]. Disponível em: http://www. cofen.gov.br/enfermagem-em-numeros.

13. Gomes HM, Marins HO. A ação docente da educação profissional São Paulo: Senac; 2004. p. 97-8.

14. Ministérios da Educação. Referências curriculares nacionais da educação profissional de nível técnico [Internet]. Brasília: 2000 [citado 2018 Nov 12]. Disponível em: http://portal.mec.gov.br/setec/arquivos/ pdf/introduc.pdf

15. Belfor JA, Sena IS, Silva DKB, Lopes BRS, Koga Júnior M, Santos BÉF. Competências pedagógicas docentes sob a percepção de alunos de medicina de universidade da Amazônia brasileira. Ciênc. Saúde Coletiva. 2018;23(1):73-82. http://dx.doi.org/10.1590/141381232018231.21342017. PMid:29267813.

16. Alberton JA, Dariff MST, Vendruscolo C, Kloh D, Zanatta EA, Pereira I. Competências necessárias ao docente em cursos de graduação em enfermagem: revisão integrativa. Rev Educ. 2017;12(2):111-22.

17. Resolução n. 466 de 12 dezembro 2012 (BR). Diretrizes e normas regulamentadoras sobre pesquisa envolvendo seres humanos. Diário Oficial da União, Brasília (DF), 12 dez 2012.

18. Thompson JE. Competencies for midwifery teachers. Midwifery 2002;18(4):256-9. http://dx.doi.org/10.1054/midw.2002.0329. PMid: 12473440.

19. Draganov PB, Friedländer MR, Sanna MC. Andragogia na saúde estudo bibliométrico. Esc Anna Nery. 2011;15(1):149-56. http://dx.doi. org/10.1590/S1414-81452011000100021.

20. Cucick CD, Faleiros F, Goes FSN, Käppler C, Pontes FAR, Silva SSC Uso de questionário online e divulgação virtual como estratégia de coleta de dados em estudos científicos. Texto Contexto Enferm. 2016;25(4):e3880014. http://dx.doi.org/10.1590/0104-07072016003880014.

21. Clarke MJ, Diguiseppi C, Edwards PJ, Kwan I, Roberts I, Wentz R et al Methods to increase response to postal and electronic questionnaires. Cochrane Database Syst Rev. 2009 jul 8;(3):MR000008. PMid:19588449.

22. Castro AE, Schuch VFJr, Vieira HC. O uso de questionários via e-mail em pesquisas acadêmicas sob a ótica dos respondentes [Internet] XIII SEMEAD - Seminários em Administração; 2010 set.; São Paulo. São Paulo: USP. [citado 5 abr 2020]. Disponível em: http://sistema.semead. com.br/13semead/resultado/trabalhosPDF/612.pdf

23. Guedes LFA, Vasconcellos L. Vantagens e Limitações dos Questionários Eletrônicos via Internet no Contexto da Pesquisa Científica [Internet] XIII SEMEAD - Seminários em Administração; 2007 ago 9-10; São Paulo. São Paulo: USP. [citado 3 abr 2020]. Disponível em: http:// sistema.semead.com.br/10semead/sistema/resultado/an_resumo. asp?cod trabalho $=420$

24. Marconi MA, Lakatos EM. Fundamentos de metodologia científica. São Paulo: Atlas, 2005. p.202.

aArtigo extraído da dissertação de mestrado intitulada: "Avaliação das competências andragógicas dos docentes do nível técnico de enfermagem", Universidade Guarulhos, 2020, de autoria de Rejane Faria Habyak Prado sob a orientação da Professora Doutora Noélle de Oliveira Freitas. 